PROCEEDINGS OF THE

AMERICAN MATHEMATICAL SOCIETY

Volume 125, Number 3, March 1997, Pages 853-860

S 0002-9939(97)03672-1

\title{
A UNIQUE CONTINUATION THEOREM FOR THE SCHRÖDINGER EQUATION WITH SINGULAR MAGNETIC FIELD
}

\author{
KAZUHIRO KURATA
}

(Communicated by Christopher D. Sogge)

\begin{abstract}
We show a unique continuation theorem for the Schrödinger equation $\left(\frac{1}{i} \nabla-\mathbf{A}\right)^{2} u+V u=0$ with singular coefficients $\mathbf{A}$ and $V$.
\end{abstract}

\section{MAIN RESUlts}

In this paper we show a unique continuation theorem for the Schrödinger operator $H=\left(\frac{1}{i} \nabla-\mathbf{A}\right)^{2}+V$ with singular magnetic field. In fact we shall establish, under some assumptions on $\mathbf{A}$ and $V$, the following estimate:

$$
\int_{B_{2 r}\left(x_{o}\right)}|u|^{2} d x \leq C \int_{B_{r}\left(x_{o}\right)}|u|^{2} d x .
$$

The latter holds for $r>0$ and $x_{o} \in \Omega$ with $B_{2 r}\left(x_{o}\right) \subset \Omega$ and for solutions $u \in$ $H_{\mathrm{loc}}^{2}(\Omega)$ of

$$
H u=\left(\frac{1}{i} \nabla-\mathbf{A}\right)^{2} u+V u=0 \quad \text { in } \quad \Omega \subset \mathbf{R}^{n},
$$

where $n \geq 3, \Omega$ is a domain, $\mathbf{A}(x)=\left(A_{j}(x)\right)_{j=1}^{n}$ is real-valued and $V(x)=$ $V^{R}(x)+i V^{I}(x)$ is complex-valued. This estimate implies a strong unique continuation theorem (Corollary 1.1). The proof uses a Rellich's type identity and the variational method (originally due to Garofalo and Lin, see e.g., [GL1], [GL2], [Ku1]), which does not need Carleman type estimates. We emphasize that our method requires neither higher integrability nor pointwise estimates for $\mathbf{A}$ compared with previous results [BKRS], [H], [Wo1], [So], [GL1], [GL2], [Ku1].

Throughout this paper we use the notation

$$
\begin{aligned}
\nabla & =\left(\frac{\partial}{\partial x_{1}}, \cdots, \frac{\partial}{\partial x_{n}}\right), \quad V^{+}(x)=\max (V(x), 0), V^{-}(x)=\max (-V(x), 0), \\
\mathbf{B} & =\left(b_{j k}\right)_{j, k=1}^{n}, \quad b_{j k}(x)=\frac{\partial A_{j}}{\partial x_{k}}-\frac{\partial A_{k}}{\partial x_{j}} \\
D_{j} & =\frac{1}{i} \frac{\partial}{\partial x_{j}}-A_{j}, \quad D_{j}^{*}=-\frac{1}{i} \frac{\partial}{\partial x_{j}}-A_{j}, \\
H_{\mathrm{loc}}^{m}(\Omega) & =\left\{u \in L_{\mathrm{loc}}^{2}(\Omega) ; \quad D^{\alpha} u \in L_{\mathrm{loc}}^{2}(\Omega),|\alpha| \leq m\right\} .
\end{aligned}
$$

Received by the editors April 3, 1995 and, in revised form, October 3, 1995.

1991 Mathematics Subject Classification. Primary 35B60, 35J10, 35Q60.

(C)1997 American Mathematical Society 
Note that $\overline{D_{j} u}=D_{j}^{*} \bar{u}$ for a complex-valued function $u$.

To state our main results, first we recall the definition of the Kato class $K_{n}^{\text {loc }}(\Omega)$.

Definition 1.1. We say $f \in L_{\text {loc }}^{1}(\Omega)$ belongs to the Kato class $K_{n}^{\text {loc }}(\Omega)$ if $\lim _{r \rightarrow 0} \eta\left(r ; f \chi_{\Omega_{o}}\right)=0$ for every compact subdomain $\Omega_{o}$ of $\Omega$. Here

$$
\eta\left(r ; f \chi_{\Omega_{o}}\right)=\sup _{x \in \mathbf{R}^{n}} \int_{\{|x-y|<r\} \cap \Omega_{o}} \frac{|f(y)|}{|x-y|^{n-2}} d y .
$$

The assumptions on $\mathbf{A}$ and $V$ in this paper are the following.

Assumption (A): Let $x_{o} \in \Omega$ be fixed.

1. $\mathbf{A} \in L_{\text {loc }}^{4}(\Omega), \quad \nabla \mathbf{A} \in L_{\mathrm{loc}}^{2}(\Omega), \quad|\mathbf{A}|^{2} \in K_{n}^{\mathrm{loc}}(\Omega), \quad\left(\left|x-x_{o}\right||\mathbf{B}|\right)^{2} \in K_{n}^{\mathrm{loc}}(\Omega)$;

2. $V \in K_{n}^{\text {loc }}(\Omega), \quad\left(\left|x-x_{o} \| V^{I}\right|\right)^{2} \in K_{n}^{\text {loc }}(\Omega),\left(2 V^{R}+\left(x-x_{o}\right) \cdot \nabla V^{R}\right)^{-} \in K_{n}^{\text {loc }}(\Omega)$.

Taking an arbitrary compact subdomain $\Omega_{o} \subset \Omega$ such that $x_{o} \in \Omega_{o}$, we will use the notation

$$
\begin{aligned}
\theta_{o}(r)= & \eta_{o}\left(r ;\left(2 V^{R}+\left(x-x_{o}\right) \cdot \nabla V^{R}\right)^{-}\right) \\
& +\eta_{o}\left(r ;\left(\left(x-x_{o}\right) V^{I}\right)^{2}\right)^{1 / 2}+\eta_{o}\left(r ;\left(\left(x-x_{o}\right)|\mathbf{B}|\right)^{2}\right)^{1 / 2},
\end{aligned}
$$

where $\eta_{o}(r ; f)=\eta\left(r ; f \chi_{\Omega_{o}}\right)$.

Theorem 1.1. Suppose Assumption (A) and $\int_{0}^{r_{o}} \frac{\theta_{o}(r)}{r} d r<+\infty$ for some $r_{o}>0$. Let $u \in H_{\mathrm{loc}}^{2}(\Omega)$ be a solution of (1). Then there exist constants $r_{*}, C>0$ such that

$$
\int_{B_{2 r}\left(x_{o}\right)}|u|^{2} d x \leq C \int_{B_{r}\left(x_{o}\right)}|u|^{2} d x
$$

for every $0<r<r_{*}$.

When we do not assume $\int_{0}^{r_{o}} \frac{\theta_{o}(r)}{r} d r<+\infty$, we have

Theorem 1.2. Suppose Assumption (A) and let $u \in H_{\mathrm{loc}}^{2}(\Omega)$ be a solution of (1). Then, for each $r_{1} \in\left(0, r_{*}\right)$, there exist constants $C>0$ and $L\left(r_{1}\right)>0$ such that

$$
\int_{B_{2 r}\left(x_{o}\right)}|u|^{2} d x \leq C \exp \left(\frac{L\left(r_{1}\right)}{r^{C \theta_{o}\left(r_{1}\right)}}\right) \int_{B_{r}\left(x_{o}\right)}|u|^{2} d x
$$

for every $r_{1} / 2>r>0$.

The constant $r_{*}$ is determined by the condition that $\eta\left(r ;\left(V^{R}\right)^{-}\right) \leq \frac{1}{2 C(n)}$ hold for all $r \in\left(0, r_{*}\right)$, where $C(n)$ is a constant depending only on $n$ (Lemma 2.3). The constants $C$ and $L\left(r_{1}\right)$ do not depend on $r$, but depend on $u$. It is well known that these yield unique continuation theorems (e.g., [GL1], [GL2], [Ku1]).

Corollary 1.1. Suppose Assumption (A) and the condition $\int_{0}^{r_{o}} \frac{\theta_{o}(r)}{r} d r<+\infty$, $r_{o}>0$, for every $x_{o} \in \Omega$. Then $H$ has $S U C P$ (strong unique continuation property); if $u \in H_{\mathrm{loc}}^{2}(\Omega)$ is a solution of (1) and satisfies, for some $x_{o} \in \Omega$ and for every $m>0$,

$$
\int_{B_{r}\left(x_{o}\right)}|u|^{2} d x=O\left(r^{m}\right) \quad(r \rightarrow 0),
$$

then $u \equiv 0$ in $\Omega$. 
Corollary 1.2. Suppose Assumption (A) for every $x_{o} \in \Omega$. Then $H$ has a unique continuation property; if $u \in H_{\mathrm{loc}}^{2}(\Omega)$ is a solution of (1) and satisfies, for some $x_{o} \in \Omega$ and $A, \alpha>0$,

$$
\int_{B_{r}\left(x_{o}\right)}|u|^{2} d x=O\left(\exp \left(-\frac{A}{r^{\alpha}}\right)\right) \quad(r \rightarrow 0),
$$

then $u \equiv 0$ in $\Omega$.

In particular, $H$ has WUCP (weak unique continuation property); if u vanishes on a subdomain $\Omega^{\prime}$ of $\Omega$, then $u \equiv 0$ in $\Omega$.

Since $H u=-\Delta u+2 i \mathbf{A} \cdot \nabla u+i(\operatorname{div} \mathbf{A}) u+|\mathbf{A}|^{2} u+V u$, putting $\mathbf{b}=2 i \mathbf{A}, W=$ $i(\operatorname{div} \mathbf{A})+|\mathbf{A}|^{2}+V$, we can rewrite the equation (1) in the following way:

$$
H u=-\Delta u+\mathbf{b} \cdot \nabla u+W u .
$$

Although we can apply results of [BKRS], [Wo1], [Wo2], [So], [H] [GL2], [Ku1] etc. under suitable conditons on $\mathbf{b}$ and $W$, our theorems cannot be covered by these previous results. First, previous results require a stronger condition on $\operatorname{div} \mathbf{A}$ even for WUCP. For instance, [Wo2] require $W \in L_{\text {loc }}^{n / 2}$ for WUCP and hence $\operatorname{div} \mathbf{A} \in L_{\text {loc }}^{n / 2}$ etc. On the other hand, our method only requires $\operatorname{div} \mathbf{A} \in L_{\text {loc }}^{2}$ for $\operatorname{div} \mathbf{A}$ and, instead of that, $\left(\left|x-x_{o}\right||\mathbf{B}|\right)^{2} \in K_{n}^{\text {loc }}(\Omega)$ (or $\left.\left(\left|x-x_{o}\right||\mathbf{B}|\right)^{2} \in F_{t}^{\text {loc }}\right)$ for the magnetic field $\mathbf{B}$ (see Example 1.1). Secondly, to obtain SUCP [BKRS], [H] require $|\mathbf{A}| \in L_{\text {loc }}^{q}, q>\frac{3 n-2}{2}$ (for related results see also [Wo1]) and [So], [GL2], [Ku1] require a pointwise estimate

$$
|\mathbf{A}(x)| \leq \frac{f\left(\left|x-x_{o}\right|\right)}{\left|x-x_{o}\right|}, \quad \int_{0}^{r_{o}} \frac{f(t)}{t} d t<+\infty
$$

for $\mathbf{A}$, but our method requires neither higher integrability nor pointwise estimates.

Remark 1.1. These theorems also hold even if we replace the class $K_{n}^{\text {loc }}(\Omega)$ in Assumption (A) by the more general one $Q_{t}^{\text {loc }}(\Omega)=K_{n}^{\text {loc }}(\Omega)+F_{t}^{\text {loc }}(\Omega), 1<t \leq n / 2$, where $F_{t}^{\text {loc }}(\Omega)$ is the Fefferman-Phong class. However, in this case we must assume an additional condition

$$
\lim _{r \rightarrow 0} \sup _{x \in \Omega}\left\|\left(V^{R}\right)^{-}\right\|_{Q_{t}\left(B_{r}(x) \cap \Omega\right)} \leq \epsilon_{o}
$$

for sufficiently small $\epsilon_{o}>0$ and take $H_{\mathrm{loc}}^{2}(\Omega) \cap L_{\mathrm{loc}}^{\infty}(\Omega)$ as a solution class. See [Ku1].

Remark 1.2. A sufficient condition to assure $\int_{0}^{r_{o}} \frac{\theta_{o}(r)}{r} d r<+\infty$ is that

$$
\left(2 V^{R}+\left(x-x_{o}\right) \cdot \nabla V^{R}\right)^{-},\left(\left(x-x_{o}\right) V^{I}\right)^{2},\left(\left(x-x_{o}\right)|\mathbf{B}|\right)^{2} \in K_{n, \delta}^{\text {loc }}(\Omega)\left(\subset K_{n}^{\text {loc }}(\Omega)\right)
$$

hold for some $\delta>0$, where we say $f \in K_{n, \delta}^{\text {loc }}(\Omega)$ if $f$ satisfies, for every compact subdomain $\Omega_{o}$ of $\Omega$,

$$
\lim _{r \rightarrow 0} \sup _{x \in \mathbf{R}^{n}} \int_{\{|x-y|<r\} \cap \Omega_{o}} \frac{|f(y)|}{|x-y|^{n-2+\delta}} d y=0 .
$$

Remark 1.3. By using the approximation argument in [Ku1] we can show unique continuation theorems similar to the theorems above even for $H_{\mathrm{loc}}^{1}$ solutions.

Finally, we must remark that Kalf [Ka] proved WUCP under the assumptions A $\in H_{\text {loc }}^{1}(\Omega),\left(V^{R}\right)^{2} \in K_{n}^{\text {loc }}(\Omega)\left(V^{I} \equiv 0\right)$, but his method cannot be applied to SUCP. For WUCP our results complement his result; compare that one needs 
$\left(V^{R}\right)^{2} \in K_{n}^{\text {loc }}(\Omega)$ in his case and $\left(2 V^{R}+\left(x-x_{o}\right) \cdot \nabla V^{R}\right)^{-} \in K_{n}^{\text {loc }}(\Omega)$ in our case (see Examples 1.2, 1.3).

Example 1.1. Let $A_{i}=C \frac{x_{i}}{|x|}$. Then $\mathbf{B} \equiv 0$ and $\nabla \mathbf{A} \in L_{\text {loc }}^{2}, \mathbf{A} \in L_{\text {loc }}^{4}$ when $n \geq 5$. Note that $|\mathbf{A}|^{2}=C^{2} /|x|^{2} \notin K_{n}^{\text {loc }}$, but $|\mathbf{A}|^{2}=C^{2} /|x|^{2} \in F_{t}^{\text {loc }}$ for $1<t<n / 2$. Hence we can apply our method to show SUCP for this A (see Remark 1.1).

Example 1.2. Let $x_{o}=O, B_{1}=B_{1}(O)$ and

$$
V(x)=\frac{1}{\left.|x|^{l}|\log | x\right|^{m}}, \quad m, l>0 .
$$

Then we have

$$
\begin{aligned}
V & \in K_{n}^{\mathrm{loc}}\left(B_{1}\right) \Leftrightarrow(m>1, l=2) \text { or }\left(m \in \mathbf{R}^{1}, l<2\right), \\
V^{2} \in K_{n}^{\mathrm{loc}}\left(B_{1}\right) & \Leftrightarrow(m>1 / 2, l=1) \text { or }\left(m \in \mathbf{R}^{1}, l<1\right), \\
2 V+x \cdot \nabla V & \in K_{n}^{\mathrm{loc}}\left(B_{1}\right) \Leftrightarrow(m>0, l=2) \text { or }\left(m \in \mathbf{R}^{1}, l<2\right) .
\end{aligned}
$$

So in this example the condition $V^{2} \in K_{n}^{\text {loc }}(\Omega)$ is stronger than $(2 V+x \cdot \nabla V)^{-} \in$ $K_{n}^{\text {loc }}(\Omega)$.

Example 1.3. Let $N \in \mathbf{N}$ and $R=\left(R_{1}, \cdots, R_{N}\right) \in \mathbf{R}^{3 N}$ be fixed and

$$
V(x)=\sum_{j=1}^{N} \frac{1}{\left|x_{j}-R_{j}\right|}, \quad x=\left(x_{1}, \cdots, x_{N}\right) \in \mathbf{R}^{3 N}, \quad x_{j} \in \mathbf{R}^{3} .
$$

Then $V^{2} \notin K_{n}^{\text {loc }}$, but $2 V+\left(x-x_{o}\right) \cdot \nabla V \in K_{n}^{\text {loc }}$ for every $x_{o} \in \mathbf{R}^{3 N}$.

\section{Proof of the THEOREMS}

We may assume $x_{o}$ is the origin $O$ and write $B_{r}=B_{r}(O)$. For the sake of simplicity, we also use $\Omega, \eta(r ; f)$ and $\theta(r)$ instead of $\Omega_{o}, \eta\left(r ; f \chi_{\Omega_{o}}\right)$ and $\theta_{o}(r)$, respectively.

Let $u \in H_{\mathrm{loc}}^{2}(\Omega)$ be a solution of (1) and put

$$
\begin{aligned}
I(r) & =\int_{B_{r}}|D u|^{2}+V^{R}|u|^{2} d x=\int_{B_{r}}|(\nabla-i \mathbf{A}) u|^{2}+V^{R}|u|^{2} d x, \\
H(r) & =\int_{\partial B_{r}}|u|^{2} d S, \quad N(r)=\frac{r I(r)}{H(r)} .
\end{aligned}
$$

Note that $H=\left(\frac{1}{i} \nabla-\mathbf{A}\right)^{2}+V=\sum_{j=1}^{n} D_{j} D_{j}+V$. Our argument is based on the following identity.

Lemma 2.1. Suppose Assumption (A) (for A). Then $u \in H_{\mathrm{loc}}^{2}(\Omega) \cap L_{\mathrm{loc}}^{\infty}(\Omega)$ satisfies

$$
\begin{aligned}
& \operatorname{Im}\left(\int_{B_{r}}(x \cdot \overline{D u}) \sum_{j=1}^{n} D_{j} D_{j} u d x\right) \\
= & \frac{r}{2} \int_{\partial B_{r}}|D u|^{2} d S-\left(\frac{n-2}{2}\right) \int_{B_{r}}|D u|^{2} d x \\
- & r \int_{\partial B_{r}}|n \cdot D u|^{2} d S+\operatorname{Re}\left(\sum_{j, k=1}^{n} \int_{B_{r}} b_{j k}(x) x_{j} D_{k} u \bar{u} d x\right)
\end{aligned}
$$

for every $r>0$ with $B_{r} \subset \Omega$. 
Proof. This is a kind of Rellich's identity. The detailed computation can be seen in [EK]. So we omit it.

We remark that under the assumption $|\mathbf{A}|^{2}, V \in K_{n}^{\text {loc }}(\Omega)$ a solution $u \in H_{\text {loc }}^{1}(\Omega)$ is locally bounded (see [Ku2]). So we can apply Lemma 2.1 for solutions $u \in H_{\mathrm{loc}}^{2}(\Omega)$ of (1). This identity implies

Lemma 2.2. Suppose Assumption (A). Then for a.e. $r \in\left(0, R_{o}\right)$

$$
\begin{aligned}
I^{\prime}(r) & =\frac{n-2}{r} I(r)+2 \int_{\partial B_{r}}|n \cdot D u|^{2} d S \\
& -\frac{n-2}{r} \int_{B_{r}} V^{R}|u|^{2} d x+\int_{\partial B_{r}} V^{R}|u|^{2} d S \\
& -\frac{2}{r} \operatorname{Im}\left(\int_{B_{r}}(x \cdot \overline{D u}) V u d x\right) \\
& -\frac{2}{r} \operatorname{Re}\left(\sum_{j, k=1}^{n} \int_{B_{r}} b_{j k}(x) x_{j}\left(D_{k} u\right) \bar{u} d x\right)
\end{aligned}
$$

holds, where $R_{o}=\max \left\{r ; B_{r} \subset \Omega\right\}$.

Proof. Note that

$$
I^{\prime}(r)=\int_{\partial B_{r}}|D u|^{2}+V^{R}|u|^{2} d S \quad \text { a.e. } r .
$$

Lemma 2.1 and this identity imply (9).

Since $x \cdot \overline{D u}=x \cdot D^{*} \bar{u}=i(x \cdot \nabla \bar{u})-(x \cdot \mathbf{A}) \bar{u}$, we have

$$
\operatorname{Im}\left(\int_{B_{r}}(x \cdot \overline{D u}) V u d x\right)=\operatorname{Re}\left(\int_{B_{r}}(x \cdot \nabla \bar{u}) V^{R} u d x\right)+\operatorname{Im}\left(\int_{B_{r}}(x \cdot \overline{D u}) V^{I} u d x\right) .
$$

Put

$$
\begin{aligned}
J\left(V^{R} ; u ; r\right) & =\int_{\partial B_{r}} V^{R}|u|^{2} d S-\frac{n-2}{r} \int_{B_{r}} V^{R}|u|^{2} d x \\
& -\frac{1}{r} \int_{B_{r}}\left(x \cdot \nabla\left(|u|^{2}\right)\right) V^{R} d x .
\end{aligned}
$$

By integration by parts we have

$$
J\left(V^{R} ; u ; r\right) \geq-\frac{1}{r} \int_{B_{r}}\left(2 V^{R}+x \cdot \nabla V^{R}\right)^{-}|u|^{2} d x .
$$

Then from this observation and Lemma 2.2 we obtain the following estimate:

$$
\begin{aligned}
I^{\prime}(r) & \geq \frac{n-2}{r} I(r)+2 \int_{\partial B_{r}}|n \cdot D u|^{2} d S \\
& -\frac{1}{r} \int_{B_{r}}\left(2 V^{R}+x \cdot \nabla V^{R}\right)^{-}|u|^{2} d x \\
& -\frac{2}{r} \int_{B_{r}}\left(|x|\left|V^{I}\right|+|x||\mathbf{B}|\right)|D u||u| d x
\end{aligned}
$$

for a.e. $r \in\left(0, R_{o}\right)$. To control the last two terms we need the following inequality. 
Lemma 2.3. Let $W \in K_{n}^{\mathrm{loc}}(\Omega)$ and $u \in H_{\mathrm{loc}}^{1}(\Omega)$. Then there exists a constant $C(n)$ such that

$$
\int_{B_{r}}|W||u|^{2} d x \leq C(n) \eta\left(r ; W \chi_{\Omega_{o}}\right)\left(\int_{B_{r}}|D u|^{2} d x+\frac{1}{r} \int_{\partial B_{r}}|u|^{2} d S\right)
$$

for $B_{r} \subset \Omega_{o}, \overline{\Omega_{o}} \subset \Omega$.

Proof. Since $|u| \in H_{\mathrm{loc}}^{1}(\Omega)$, we know that

$$
\int_{B_{r}}|W||u|^{2} d x \leq C(n) \eta\left(r ; W \chi_{\Omega_{o}}\right)\left(\left.\int_{B_{r}}|\nabla| u\right|^{2} d x+\frac{1}{r} \int_{\partial B_{r}}|u|^{2} d S\right)
$$

holds( [FGL]). Noting $|\nabla| u|| \leq|D u|$ a.e., we obtain the desired inequality.

Lemma 2.4. There exists a constant $r_{*}>0$ such that $H(r)>0$ for every $r \in$ $\left(0, r_{*}\right)$ unless $u \equiv 0$ in $B_{r_{*}}$.

Proof. Note that the constant $r_{*}>0$ is determined by the condition $\eta_{-}^{R}(r) \leq \frac{1}{2 C(n)}$ for $r \in\left(0, r_{*}\right)$, where $\eta_{-}^{R}(r)=\eta\left(r ;\left(V^{R}\right)^{-}\right)$and $C(n)$ is the constant in Lemma 2.3. Since $V^{R} \in K_{n}^{\text {loc }}(\Omega)$, the constant $r_{*}>0$ satisfying this condition exists.

Suppose $H\left(r_{o}\right)=0$ for some $r_{o} \in\left(0, r_{*}\right)$. Noting a simple conputation yields

$$
I(r)=\operatorname{Re}\left(\int_{\partial B_{r}} u_{\rho} \bar{u} d S\right)=\operatorname{Re}\left(\int_{\partial B_{r}}(n \cdot D u) \bar{u} d S\right),
$$

we have $I\left(r_{o}\right)=0$. Since Lemma 2.3 implies

$$
\begin{aligned}
I(r) & \geq \int_{B_{r}}|D u|^{2} d x-\int_{B_{r}}\left(V^{R}\right)^{-}|u|^{2} d x \\
& \geq \int_{B_{r}}|D u|^{2} d x-C(n) \eta_{-}^{R}(r)\left(\frac{H(r)}{r}+\int_{B_{r}}|D u|^{2} d x\right),
\end{aligned}
$$

the choice of $r_{*}$ yields

$$
0=I\left(r_{o}\right) \geq \frac{1}{2} \int_{B_{r_{o}}}|D u|^{2} d x .
$$

Hence we obtain $|D u(x)|=0$ a.e. $x \in B_{r_{o}}$. Since $|\nabla| u|| \leq|D u|$ a.e., $|u|$ is constant in $B_{r_{o}} . H\left(r_{o}\right)=0$ implies $|u| \equiv 0$ in $B_{r_{o}}$. The conclusion can be obtained by an argument similar to the one in [Ku1, Theorem 1.5].

Hence we may assume $H(r)>0$ for every $r \in\left(0, r_{*}\right)$.

Lemma 2.5. There exists an absolute constant $C_{0}>0$ such that

$$
\int_{B_{r}}|D u|^{2} d x \leq C_{0} I(r)
$$

for every $r \in \Gamma=\left\{r \in\left(0, r_{*}\right) ; N(r)>1\right\}$.

Proof. Since $\frac{H(r)}{r}<I(r)$ for $r \in \Gamma,(15)$ implies

$$
\int_{B_{r}}|D u|^{2} d x \leq \frac{1+C(n) \eta_{-}^{R}(r)}{1-C(n) \eta_{-}^{R}(r)} I(r) \leq 3 I(r)
$$

for all $r \in \Gamma$. This concludes the desired estimate. 
Using (11) and Lemmas 2.3, 2.5 we obtain, for a.e. $r \in \Gamma$,

$$
\begin{gathered}
I^{\prime}(r) \geq \frac{n-2}{r} I(r)+2 \int_{\partial B_{r}}|n \cdot D u|^{2} d S-C \frac{\theta(r)}{r} I(r), \\
\theta(r) \eta\left(r ;\left(2 V^{R}+x \cdot \nabla V^{R}\right)^{-}\right)+\eta\left(r ;\left(|x| V^{I}\right)^{2}\right)^{1 / 2}+\eta\left(r ;(|x||\mathbf{B}|)^{2}\right)^{1 / 2} .
\end{gathered}
$$

On the other hand, we have the following identity:

$$
H^{\prime}(r)=\frac{n-1}{r} H(r)+2 \operatorname{Re}\left(\int_{\partial B_{r}} u_{\rho} \bar{u} d S\right), \quad u_{\rho}=\frac{x}{r} \cdot \nabla u .
$$

Hence (14) and (19) imply

$$
\begin{aligned}
H^{\prime}(r) & =\frac{n-1}{r} H(r)+2 I(r), \\
\frac{d}{d r}\left(\log \frac{H(r)}{r^{n-1}}\right) & =2 \frac{I(r)}{H(r)}=2 \frac{N(r)}{r} .
\end{aligned}
$$

Therefore, for a.e. $r \in \Gamma$ we obtain

$$
\begin{aligned}
\frac{N^{\prime}(r)}{N(r)} & =\frac{1}{r}+\frac{I^{\prime}(r)}{I(r)}-\frac{H^{\prime}(r)}{H(r)} \\
& \geq 2\left(\frac{\int_{\partial B_{r}}|n \cdot D u|^{2} d S}{I(r)}-\frac{I(r)}{H(r)}\right)-C \frac{\theta(r)}{r} \\
& \geq-C \frac{\theta(r)}{r} .
\end{aligned}
$$

In the last inequality we used Schwarz's inequality. This differential inequality yields the following growth estimate for $N(r)$.

Theorem 2.1. (i) If $\int_{0}^{r_{o}} \frac{\theta(r)}{r} d r<+\infty$ for some $r_{o}>0$, then

$$
N(r) \leq \max \left(1, N\left(r_{*}\right)\right) \exp \left(C \int_{0}^{r_{*}} \frac{\theta(r)}{r} d r\right)
$$

for every $r \in\left(0, r_{*}\right)$.

(ii)

In general, for each $r_{1} \in\left(0, r_{*}\right)$ there exist constants $C, L\left(r_{1}\right)>0$ such that

$$
N(r) \leq \frac{L\left(r_{1}\right)}{r^{C \theta\left(r_{1}\right)}}
$$

for every $r \in\left(0, r_{1} / 2\right)$.

This theorem implies Theorems 1.1 and 1.2 by a standard argument. For the details see [Ku1], [GL2].

\section{ACKNOWLEDGEMENT}

I would like to thank Professor J. Uchiyama for informing me of the paper [Ka]. 


\section{REFERENCES}

[BKRS] Barcelo, B., Kenig, C. E., Ruiz, A., Sogge, C. D. Weighted Sobolev inequalities and unique continuation for the Laplacian plus lower order terms. Illinois J.Math. 32(1988), 230-245. MR 89h:35048

[EK] Eastham, M. S. P., Kalf, H. Schrödinger-type operators with continuous spectra. Pitman Lecture Note Series 65, Longman. MR 84i:35107

[FGL] Fabes, E. B., Garofalo, N., Lin, F. H. A partial answer to a conjecture of B.Simon concerning unique continuation. J. Fun. Ana. 88(1990), 194-210. MR 91m:35074

[GL1] Garofalo, N., Lin, F. H. Monotonicity properties of variational integrals, $A_{p}$ weights and unique continuation. Indiana Univ. Math. J. 35(1986), 245-268. MR 88b:35059

[GL2] Garofalo, N., Lin, F. H. Unique continuation for elliptic operators: A geometricvariational approach. Comm. Pure Appl. Math. 40(1987), 347-366. MR 88j:35046

[Ka] Kalf, H. Une remarque au sujet de prolongement unique des solutions de l'équation de Schrödinger. C.R. Acad. Sc. Paris, 295(1982), 579-581. MR 84f:35032

[H] Hörmander, L. Uniqueness theorems for second-order elliptic differential equations. Comm. in P.D.E. 8(1983), 21-64. MR 85c:35018

[Ku1] Kurata, K. A unique continuation theorem for uniformly elliptic equations with strongly singular potentials. Comm. in P.D.E. 18(7\& 8) (1993), 1161-1189. MR 95b:35034

[Ku2] Kurata, K. Local boundedness and continuity for weak solutions of $-(\nabla-i \mathbf{b})^{2} u+V u=0$. Math. Z. (to appear).

[So] Sogge, C. D. Strong uniqueness theorems for second order elliptic differential equations. Amer. J. Math. 112(1990), 943-984. MR 91k:35068

[Wo1] Wolff, T. H. Unique continuation for $|\Delta u| \leq V|\nabla u|$ and related problems. Revista Math. Iberoamericana 6(1990), 155-200. MR 93a:35061

[Wo2] Wolff, T. H. A property of measures in $\mathbf{R}^{n}$ and an application to unique continuation. Geometric and Fun. Ana. 2, No. 2 (1992), 225-284. MR 93c:35015

Department of Mathematics, Tokyo Metropolitan University, Minami-Ohsawa 1-1, HACHIOJI-SHI, TOKYO, 192-03 JAPAN

E-mail address: kurata@math.metro-u.ac.jp 